\title{
miR-26a promotes neurite outgrowth by repressing PTEN expression
}

\author{
BAOGUO LI and HANXIAO SUN
}

Institute of Genomic Medicine, College of Pharmacy, Jinan University, Guangzhou, Guangdong 510632, P.R. China

Received April 10, 2013; Accepted June 3, 2013

DOI: $10.3892 / \mathrm{mmr} .2013 .1534$

\begin{abstract}
A multitude of studies have reported that microRNAs (miRNAs) are important in neuronal development. As a key regulator altering the expression of certain mRNAs, miR-26a has been demonstrated to play a role in the central nervous system (CNS). In the current study, the function of miR-26a in neuronal development was investigated. The overexpression of miR-26a was hypothesized to significantly enhance synaptic plasticity and regulate neuronal morphogenesis. The number and distribution of neurites was markedly increased by miR-26a. In addition, inhibition of miR-26a function attenuated neuronal outgrowth. Furthermore, phosphatase and tensin homolog (PTEN) was identified as a direct target of miR-26a in this process via a luciferase reporter assay. The growth of neurites was consistently suppressed by PTEN overexpression. Therefore, our study demonstrated that miR-26a promoted neurite outgrowth via the suppression of PTEN expression, indicating that miR-26a is important in neuronal development and morphogenesis. miR-26a has the potential to serve as a therapeutic target for patients with Alzheimer's disease (AD).
\end{abstract}

\section{Introduction}

Alzheimer's disease (AD) is the most common dementing illness globally. By 2050, one person in every 85 is predicted to suffer from this disease (1). Neurodegenerative diseases may cause memory loss, impaired cognitive function and eventually, death. Pathological features include the impairment of axonal and dendritic transport, swelling of axons, dendrites and varicosities and breakage. Early experiments revealed that the maintenance of normal neurite transport and the morphology of neurons are vital for the biological functions of neurons (2). Our study casts light on the mechanism

Correspondence to: Professor Hanxiao Sun, Institute of Genomic Medicine, College of Pharmacy, Jinan University, 601 Huangpu Street, Guangzhou, Guangdong 510632, P.R. China

E-mail: sunhx718@yahoo.com.cn

Key words: Alzheimer's disease, miR-26a, neurite outgrowth, phosphatase and tensin homolog of neuronal development and provides a novel and valuable possibility for therapeutic intervention in AD.

Gene modulation, particularly post-transcriptional gene regulation, acts as a pivotal regulator in brain development (3). MicroRNAs (miRNAs) are a type of non-coding small RNA mediating gene silencing, and are 21- to 23-nucleotides long. Thousands of miRNAs have been identified in mammals, a number of which are expressed spatially and temporally in the process of brain development (4). Significant progress has been made in identifying targets of miRNAs. More than $60 \%$ of all mammalian mRNAs are tightly controlled by miRNAs. miRNAs are part of signaling networks serving as an important mediator of crosstalk between signaling pathways, coordinating their activity $(4,5)$. miRNA biogenesis pathways are widely recognized as regulators in the development of the central nervous system (CNS). For instance, miR-124, which accounts for $25-48 \%$ of all brain-specific miRNAs, has been studied extensively (6). miR-9 is specifically and abundantly expressed in the neurogenic regions of the brain (7). miR-124 and miR-128 are prone to appear in neurons, whereas miR-23 is expressed in astrocytes. miR-132 has been reported to modulate neuronal morphogenesis via suppressing the expression of the GTPase-activating protein p250GAP (8). miR-134 has been reported to regulate the development of neurite spines through repressing expression of the Limk1 protein kinase (9). During brain development, the expression of miR-128 is upregulated, and ectopic expression of miR-128 may promote neural differentiation and augment the average dendritic spine length of neural stem cells (NSCs) (10). Accumulating evidence supports the crucial roles of miRNAs in neuronal development. Our current study demonstrated that the expression of miR-26a in neurons is higher than that in NSCs; however, the function of miR-26a in neurons has not been validated experimentally. In addition, the correlation between miR-26a, synaptic plasticity and the growth of neurites has not yet been documented. Therefore, the present study focused on the function of miR-26a in neuronal outgrowth.

\section{Materials and methods}

Cell culture and transfection. All procedures were performed in accordance with a protocol approved by the Institutional Animal Care and Use Committee (Chinese Academy of Science). Neonatal rat cortical neurons and NSCs were obtained from the cortex of male Sprague-Dawley rats (purchased from 
the Animal Center of Chinese Academy of Science, Beijing, China), as described previously (11). Neurons were plated on poly-D-lysine $(10 \mu \mathrm{g} / \mathrm{ml})$ and laminin $(5 \mu \mathrm{g} / \mathrm{ml}$; Sigma-Aldrich, St. Louis, MO, USA) -coated glass. The electroporation of primary cortical neurons was conducted using a rat neuron nucleofector kit (Amaxa Biosystems, Gaithersburg, MD, USA) according to the manufacturer's instructions, and the neurons were cultured in neurobasal media supplemented with factor B27, $2 \mathrm{mM}$ L-glutamine, $0.06 \mathrm{mg} / \mathrm{ml}$ cysteine, $1 \mathrm{mM}$ sodium pyruvate, penicillin and streptomycin (Invitrogen, Carlsbad, $\mathrm{CA}, \mathrm{USA}$ ) at $37^{\circ} \mathrm{C}$ and $5 \% \mathrm{CO}_{2}$.

Expression vectors. The phosphatase and tensin homolog (PTEN) and its short hairpin RNA (shRNA) were amplified and cloned into pCAG-EGFP. The primers were as follows: PTEN forward, 5'-CGGGATCCGACATGACAGCCATCA TCAAAG-3' and reverse, 5'-CCGCTCGAGTCAGACTTTTGT AATTTGTGTATG-3'; shRNA forward, 5'-GGCGCUAUGU GUAUUAUUATT-3' and reverse, 5'-UAAUAAUACACAUAG CGCCTT-3'; and control miRNA sense, 5'-UUCUCCGAACGU GUCACGUTT-3' and anti-sense 5'-ACGUGACACGUUCGGA GAATT-3'. The sequences for the miR-26a mimics were as follows: sense, 5'-UUCAAGUAAUCCAGGAUAGGCU-3' and anti-sense, 5'-CCUAUCCUGGAUUACUUGAAUU-3'; miR-26a inhibitor, 5'-AGCCUAUCCUGGAUUACUUGAA-3' and control, 5'-CAGUACUUUUGUGUAGUACAA-3'.

Cell viability analysis. The viability of transfected neurons was determined by a CCK assay kit (Dojindo, Kumamoto, Japan). Transfected neurons were seeded at a density of $5 \times 10^{3}$ cells per well into 96-well plates. The CCK-8 solution was diluted with neuronal media (1:10), and $100 \mu \mathrm{l}$ of the resulting solution was added to each well and incubated for $2 \mathrm{~h}$ at $37^{\circ} \mathrm{C}$. The absorbance was determined at $450 \mathrm{~nm}$ by a microplate reader (BioTek, Winooski, VT, USA).

Western blot analysis. For western blot analysis, cells were first washed with cold PBS 3 times and then lysed in RIPA buffer (Solarbio, Beijing, China). Protein lysates $(30 \mu \mathrm{g})$ were electrophoresed on SDS-PAGE gels and transferred to PVDF membranes (Millipore, Billerica, MA, USA). The membranes were blocked for $1 \mathrm{~h}$ at room temperature (RT) with $5 \%$ milk protein and $0.05 \%$ Tween 20 in PBS. Mouse monoclonal anti-PTEN IgG and mouse monoclonal anti-GADPH (Cell Signaling Technology, Inc., Danvers, MA, USA) were used according to the manufacturer's instructions. The membranes were washed 3 times with PBS for $10 \mathrm{~min}$ and then incubated for $1 \mathrm{~h}$ at RT with the anti-mouse infrared dye-conjugated secondary antibodies. After washing 3 times, the bands and band intensity were analyzed using an Odyssey instrument (LI-COR, USA).

Reverse transcription and quantitative (q)PCR. Total RNA was isolated from cells using TRIzol (Invitrogen) according to the manufacturer's instructions. For miR-26a, total RNA (50 ng) was transcribed into cDNA using a TaqMan MicroRNA Reverse Transcription kit (Applied Biosystems, Carlsbad, CA, USA) according to the manufacturer's instructions. qPCR was performed with a TaqMan MicroRNA Assay Kit (Applied Biosystems). The expression of U6 was used as a control, and the fold-change was calculated by the $2^{-\Delta \Delta C t}$ method. The amplification and detection of specific products were performed using the ABI Prism 7500 system (Applied Biosystems).

Luciferase reporter assay. The 3' untranslated region (3'-UTR) sequence of rat PTEN mRNA containing the miR-26a binding site was amplified and cloned into a pMIR-REPORT luciferase reporter vector, known as pMIR-PTEN-3'UTR. The vector of PTEN 3'UTR with the miR-26a binding site was co-transfected separately with miR-26a, miRNA control or miR-26a inhibitor into primary neurons. After $24 \mathrm{~h}$, the luciferase assay was performed according to the manufacturer's instructions.

Immunocytochemistry and quantitation of neuronal morphology. Cortical neurons were fixed with $4 \%$ paraformaldehyde at RT for $30 \mathrm{~min}$. Neurons were blocked $(1 \mathrm{~h}$ at RT) in 5\% BSA in PBS with $0.01 \%$ Triton X-100 and probed with neuronal class III $\beta$-tubulin (1:1,000; Sigma-Aldrich) and DAPI $(2 \mu \mathrm{g} / \mathrm{ml}$; Sigma-Aldrich). The cells were then analyzed under a Zeiss 780 confocal microscope (Carl Zeiss AG, Oberkochen, Germany). Images of neurons were traced and quantified by the NeuronJ program (12).

Statistical analysis. All data are presented as the mean \pm standard deviation (SD). Variance analysis between the groups was performed by one-way ANOVA, and significant differences between the groups were analyzed by Dunnett's multiple comparison test. $\mathrm{P}<0.05$ was considered to indicate a statistically significant difference. All statistical analysis was performed using SPSS 16.0 statistical software (SPSS, Inc., Chicago, IL, USA).

\section{Results}

miR-26a is highly expressed in mature neurons. miR-26a has exhibited a consistent pattern of expression in in vivo and in vitro models (12). To identify whether miR-26a is involved in the development of neurons, qPCR analysis was performed to characterize its expression. The expression of miR-26a in neurons was markedly increased by 3.4-fold compared with NSCs (Fig. 1A). Thus, the expression level of miR-26a was higher in neurons than in NSCs. These data suggested a marked correlation between neuronal development and the miR-26a expression level, indicating that miR-26a may play a role in the development of neurons.

Expression of miR-26a progressively promotes the growth of neurites. Considering the expression of miR-26a in primary neurons, we examined whether miR-26a plays a role in regulating the growth of neurites. With the upregulation of miR-26a, neurite morphology significantly changed. Morphometric analysis revealed that miR-26a-transfected neurons had higher neurite numbers and the average total length was greater than that of control miRNA-transfected neurons (Fig. 1B). In addition, transfection of miR-26a inhibitor (miR-26a knockdown, KD) induced a marked decrease in the distribution of neurites and total neurite length (Fig. 1B). Growth curves of the two groups were generated and it was found that the neurite branches were more pronounced in the miR-26a over- 
A

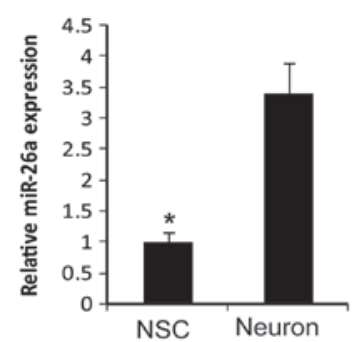

B
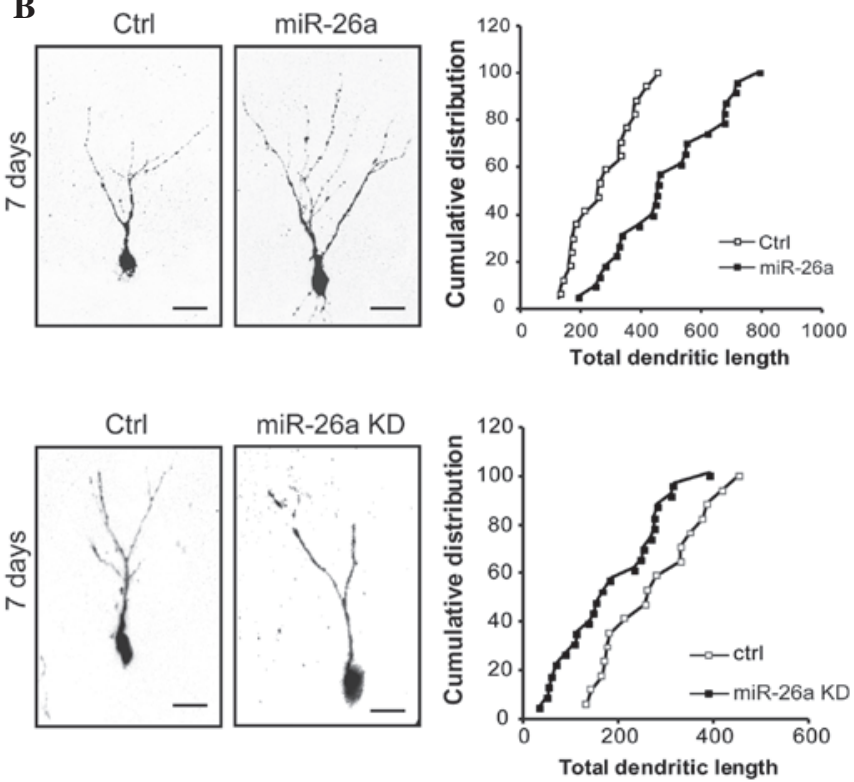

C

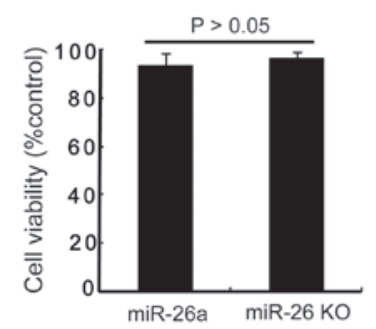

Figure 1. Expression of miR-26a in neurons regulates the growth of neurites. (A) The expression of miR-26a in NSCs and neurons was assessed by quantitative (q)PCR analysis. The abundance of miR-26a was standardized to that of U6. ${ }^{*} \mathrm{P}<0.05$ vs. the neuron group. (B) Neurons transfected with miRNA-control (Ctrl), miR-26a mimic (miR-26a) and miR-26a inhibitor (miR-26a KD) were immunostained for the neuronal specific marker $\beta$-III tubulin (TUJ1) and analyzed morphometrically. Overexpression of miR-26a significantly enhanced the outgrowth of neurites and induced widespread distribution. Knockdown of miR-26a significantly inhibited the number and outgrowth of neurites. Scale bars, $100 \mu \mathrm{m}$. (C) Neurons treated as described in (B) were collected 3 days later and analyzed by a CCK assay kit. All data were obtained from at least three independent experiments and are shown as the mean \pm standard deviation. NSCs, neural stem cells.

expression (OE) group (Fig. 1B). To further examine whether this phenotype was associated with the viability of neurons, neuronal apoptosis was measured by a CCK assay, and it was shown that miR-26a (OE) and miR-26a (KD) did not affect the survival of neurons $(94.9 \pm 4.3$ vs. $96.1 \pm 2.8 \%$; Fig. 1C). Taken together, our data suggested that miR-26a promotes the growth of neurites and does not induce cell death.

PTEN is a direct target of miR-26a in neurons. A number of genes have been reported to be the target of miR-26a. To
A

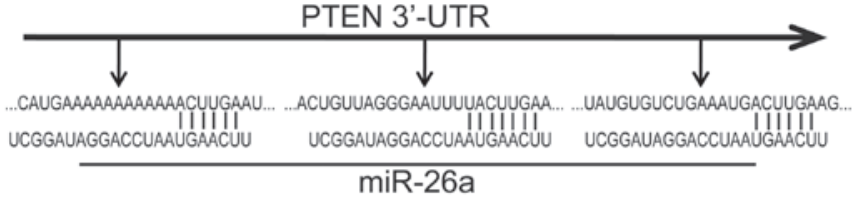

B
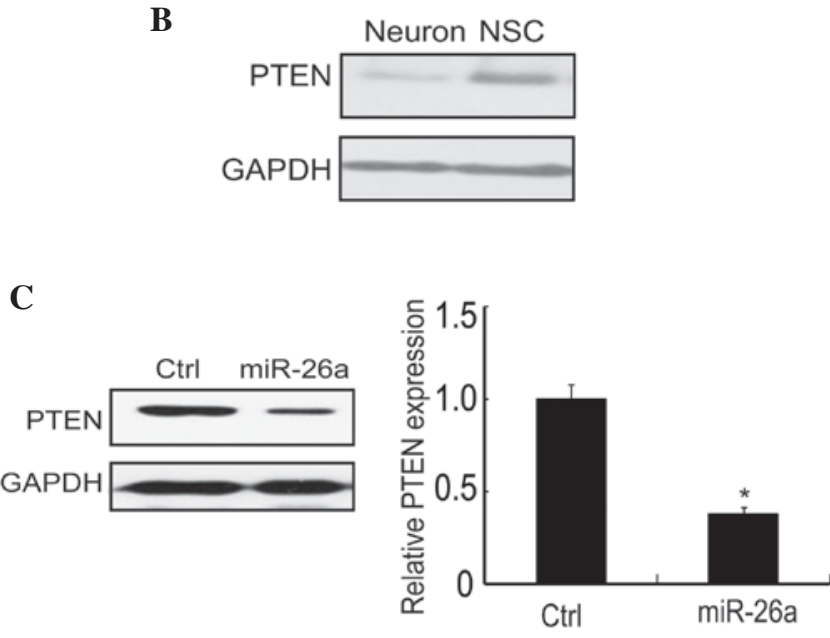

D

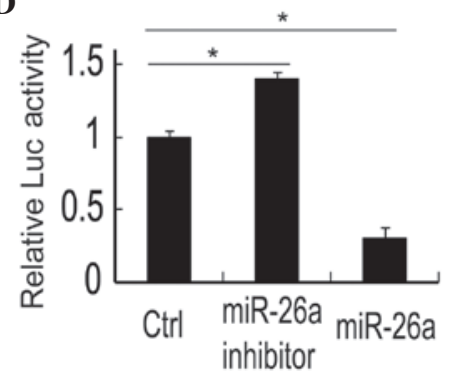

Figure 2. PTEN is a direct target of miR-26a in neurons. (A) The PTEN 3'UTR has three predicted binding sites of miR-26a. (B) The normal expression of PTEN in neurons and NSCs was detected by western blot analysis. (C) miR-26a reduced the expression of PTEN. Neurons transfected with miRNA-control (Ctrl) and miR-26a mimic (miR-26a) were collected to detect PTEN by western blot analysis after $72 \mathrm{~h}$. Similar results were obtained in three independent experiments. (D) A luciferase assay was performed as described in Materials and methods. The values are presented as the mean \pm S.D. of three independent experiments. "P<0.05. PTEN, phosphatase and tensin homolog; 3'UTR, 3' untranslated region; NSCs, neural stem cells; Luc, luciferase.

gain insight into the mechanisms by which miR-26a exerts its biological functions (13-15), we explored predictive targets of miR-26a through the TargetScan bioinformatics algorithm (www.targetscan.org). Result revealed that the 3'-UTR of PTEN has three potential binding sites, which are highly evolutionarily conserved (Fig. 2A). Due to miRNA modulation, the correspondent mRNA expression and protein level may change (6). Our data showed that the protein expression of PTEN is higher in NSCs than in neurons (Fig. 2B). On further study, the expression of PTEN protein was specifically reduced in neurons transfected with miR-26a mimics, and the dose of endogenous PTEN was reduced to $38.3 \%$ (Fig. 2C). Therefore, these results indicated that there was a close correlation between PTEN and miR-26a. A luciferase reporter gene 
A

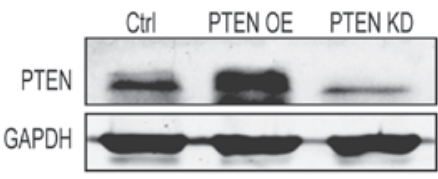

B

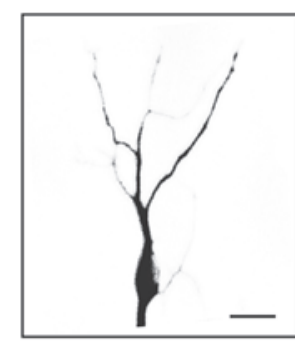

Ctrl Vector+miR control
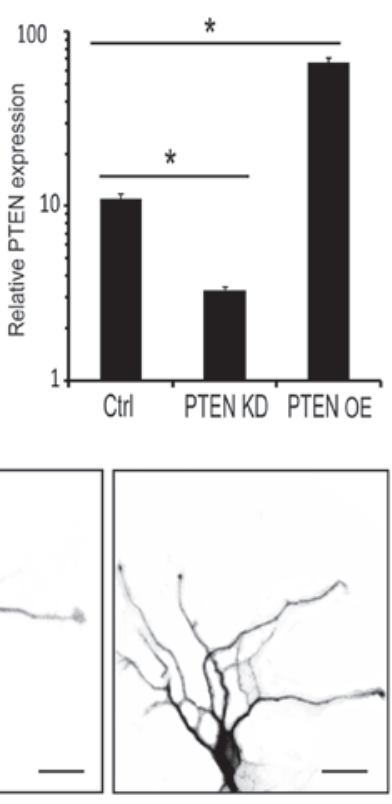

PTEN KD+miR-26a KD
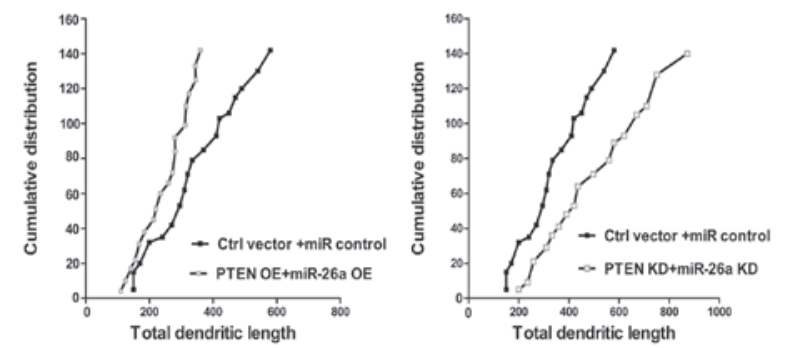

Figure 3. PTEN mediates the effect of miR-26a on neurite outgrowth. (A) Neurons treated with PTEN (PTEN OE), shRNA of PTEN (PTEN KO) and a control vector (Ctrl) were collected to detect the expression of PTEN by western blot analysis. ${ }^{\mathrm{P}}<0.05$. (B) The effect of PTEN on growth was determined by immunofluorescence staining and analyzed morphometrically. The values are presented as the mean \pm S.D. of three independent experiments. PTEN, phosphatase and tensin homolog; shRNA, short hairpin RNA.

assay was performed in order to determine whether PTEN is a direct target of miR-26a. Firstly, the 3'-UTR of PTEN with one binding site was cloned into the pMIR-REPORT luciferase vector as a positive control. This plasmid was then co-transfected with miR-26a mimic, its control miR-control and miR-26a inhibitor into primary neurons. The results indicated that luciferase activity was markedly decreased in the miR-26a group $(31 \pm 4.2 \%)$, whereas it did not change with miR-control and was increased in the miR-26a inhibitor group (139 $\pm 2.1 \%$; Fig. 2D). Taken together, these data revealed that miR-26a suppressed the expression of PTEN and that PTEN is a direct target of miR-26a.

PTEN mediates the effect of miR-26a on neurite outgrowth. miR-26a was shown to promote neurite outgrowth and PTEN is involved in this process. To further investigate the effect of PTEN on the growth of neurites, the efficiency of PTEN overexpression (PTEN OE) and its knockdown (PTEN KD) were examined (Fig. 3A). The results showed that neurons transfected with PTEN generated an opposite result compared with neurons transfected with miR-26a; the growth of neurites was largely suppressed. By contrast, transfection with the shRNA of PTEN enhanced neurite outgrowth, and the number and distribution of neurites was significantly increased (Fig. 3B). The overexpression of PTEN abolished the enhancement of neurite growth by miR-26a. PTEN knockdown rescued the growth defect of neurons induced by treatment with miR-26a inhibitor. The number and length of neurites was attenuated and suppressed by PTEN overexpression. Therefore, we concluded that PTEN is an important molecule in this signaling pathway, which has marked biological control over the development of the neuron, particularly neuronal morphogenesis.

\section{Discussion}

With the advance of therapeutics, the biodemographics of the world is likely to change, since the number of elderly people is constantly increasing. The number of patients with $\mathrm{AD}$ and related dementias will also increase significantly from current levels $(16,17)$. The cognitive impairment of patients seriously affects their quality of life (1); therefore, finding a method to cure or prevent AD and other dementias is important. Current research has provided us with more information on the basic biology of AD and this will make the prevention of AD possible (1).

$\mathrm{AD}$ is the most common neurodegenerative disease. Axonopathy and dendropathy is a subset of AD. It has been demonstrated that the normal link between neurons in the cortex is critical for the function of the brain $(18,19)$. Numerous miRNAs may also be involved in this process, including miR9, miR124, miR155 and miR132. Together, these molecules maintain the normal development of the CNS $(8,20-22)$. Our study showed that the expression of miR-26a was lower in NSCs than in neurons, and lower in dysfunctional neurons. Furthermore, we identified that miR-26a promotes the growth of neurites and increases the number and length of the neurite branch. These results indicate that miR-26a may be capable of ameliorating cognitive impairment, although this requires further demonstration in in vivo experiments. The pathway of miR-26a is considered to be different depending on the cell and tissue types. A number of previous studies have shown that PTEN is a target of miR-26a $(23,24)$. However, none of these studies involved neurons. Our study revealed that PTEN is important in neuronal development and that miR-26a affects the morphology of neurons. It is conceivable that there may be other additional targets regulated by miR-26a. This process also requires the coordinated function of several genes, including the genes associated with cytoskeletal reorganization (25). Further study is required to explore the underlying mechanisms and the details of signaling pathways involved in this process (26-28). A conditional mouse model may provide more details on the precise role of miR-26a in neurite outgrowth and neuronal maturation. These results and future work may provide a novel insight into the mechanism of neuronal dysfunction and may be beneficial for the prevention of AD.

miR-26a was found to be capable of regulating neurite outgrowth via the repression of PTEN expression. The overexpression of miR-26a may induce the growth of neurites, and 
this is beneficial to the function of impaired neurons. This finding may prove useful for the treatment of patients with AD.

\section{Acknowledgements}

This work was sponsored by the National Science Foundation of China (NSFC; no. 3087221).

\section{References}

1. Carrillo MC, Brashear HR, Logovinsky V, et al: Can we prevent Alzheimer's disease? Secondary "prevention" trials in Alzheimer's disease. Alzheimers Dement 9: 123-131, 2013.

2. McKhann G, Drachman D, Folstein M, et al: Clinical diagnosis of Alzheimer's disease. Neurology 34: 939, 1984.

3. Bartel DP: MicroRNAs: target recognition and regulatory functions. Cell 136: 215-233, 2009.

4. Inui M, Martello G and Piccolo S: MicroRNA control of signal transduction. Nat Rev Mol Cell Biol 11: 252-263, 2010.

5. He L and Hannon G J: MicroRNAs: small RNAs with a big role in gene regulation. Nat Rev Genet 5: 522-531, 2004

6. Lagos-Quintana M, Rauhut R, Yalcin A, Meyer J, Lendeckel W and Tuschl T: Identification of tissue-specific microRNAs from mouse. Curr Biol 12: 735-739, 2002.

7. Deo M, Yu JY, Chung KH, Tippens M and Turner DL: Detection of mammalian microRNA expression by in situ hybridization with RNA oligonucleotides. Dev Dyn 235: 2538-2548, 2006.

8. Vo N, Klein ME, Varlamova O, et al: A cAMP-response element binding protein-induced microRNA regulates neuronal morphogenesis. Proc Natl Acad Sci USA 102: 16426-16431, 2005.

9. Lim LP, Lau NC, Garrett-Engele P, et al: Microarray analysis shows that some microRNAs downregulate large numbers of target mRNAs. Nature 433: 769-773, 2005.

10. Bruno IG, Karam R, Huang L, et al: Identification of a microRNA that activates gene expression by repressing nonsense-mediated RNA decay. Mol Cell 42: 500-510, 2011.

11. Nikolic M, Chou MM, Lu W, Mayer BJ and Tsai LH: The $\mathrm{p} 35 / \mathrm{Cdk} 5$ kinase is a neuron-specific Rac effector that inhibits Pak1 activity. Nature 395: 194-198, 1998.

12. Meijering E and Jacob M: Design and validation of a tool for neurite tracing and analysis in fluorescence microscopy images. Cytometry A 58: 167-176, 2004.

13. Zhang YF,Zhang AR,Zhang BC, et al: miR-26a regulates cell cycle and anoikis of human esophageal adenocarcinoma cells through Rb1-E2F1 signaling pathway. Mol Biol Rep 40: 1711-1720, 2013.

14. Miko E, Czimmerer Z, Csánky E, et al: Differentially expressed microRNAs in small cell lung cancer. Exp Lung Res 35: 646-664, 2009.
15. Liu B, Wu X, Liu B, et al: miR-26a enhances metastasis potential of lung cancer cells via AKT pathway by targeting PTEN. Biochim Biophys Acta 1822: 1692-1704, 2012.

16. Kim H, Huang W, Jiang X, et al: Integrative genome analysis reveals an oncomiR/oncogene cluster regulating glioblastoma survivorship. Proc Natl Acad Sci USA 107: 2183-2188, 2010.

17. Vaupel JW: Biodemography of human ageing. Nature 464: 536-542, 2010

18. Graves AB, Larson EB, Edland SD, Bowen JD, et al: Prevalence of dementia and its subtypes in the Japanese American population of King County, Washington state. The Kame Project. Am J Epidemiol 144: 760-771, 1996.

19. Moceri VM, Kukull WA, Emanuel I, van Belle G and Larson EB: Early-life risk factors and the development of Alzheimer's disease. Neurology 54: 415-420, 2000.

20. Cheng LC, Pastrana E, Tavazoie M and Doetsch F: miR-124 regulates adult neurogenesis in the subventricular zone stem cell niche. Nat Neurosci 12: 399-408, 2009.

21. Zhao C, Sun G, Li S and Shi Y: A feedback regulatory loop involving microrna- 9 and nuclear receptor tlx in neural stem cell fate determination. Nat Struct Mol Biol 16: 365-371, 2009.

22. Dunand-Sauthier I, Santiago-Raber ML, Capponi L, et al: Silencing of c-Fos expression by microRNA-155 is critical for dendritic cell maturation and function. Blood 117: 4490-4500, 2011.

23. Lobnig BM, Krömeke O, Optenhostert-Porst C and Wolf OT: Hippocampal volume and cognitive performance in long-standing type 1 diabetic patients without macrovascular complications. Diabet Med 23: 32-39, 2006.

24. Zhang B, Liu XX, He JR, et al: Pathologically decreased miR-26a antagonizes apoptosis and facilitates carcinogenesis by targeting MTDH and EZH2 in breast cancer. Carcinogenesis 32: 2-9, 2011

25. Alajez NM, Shi W, Hui AB, et al: Enhancer of Zeste homolog 2 $(\mathrm{EZH} 2)$ is overexpressed in recurrent nasopharyngeal carcinoma and is regulated by miR-26a, miR-101, and miR-98. Cell Death Dis 1: e85, 2010

26. Kuhn TB, Meberg PJ, Brown MD, et al: Regulating actin dynamics in neuronal growth cones by $\mathrm{ADF} /$ cofilin and rho family GTPases. J Neurobiol 44: 126-144, 2000.

27. Jacobson C, Schnapp B and Banker GA: A change in the selective translocation of the Kinesin-1 motor domain marks the initial specification of the axon. Neuron 49: 797-804, 2006.

28. Jiang H, Guo W, Liang X and Rao Y: Both the establishment and the maintenance of neuronal polarity require active mechanisms: critical roles of GSK-3beta and its upstream regulators. Cell 120: $123-135,2005$ 